\title{
BENCHMARKING OF OBJECTIVE QUALITY METRICS FOR COLORLESS POINT CLOUDS
}

\author{
Evangelos Alexiou and Touradj Ebrahimi \\ Multimedia Signal Processing Group (MMSPG) \\ École Polytechnique Fédérale de Lausanne (EPFL) \\ CH-1015, Lausanne, Switzerland \\ Emails: FirstName.LastName@epfl.ch
}

\begin{abstract}
Recent advances in depth sensing and display technologies, along with the significant growth of interest for augmented and virtual reality applications, lay the foundation for the rapid evolution of applications that provide immersive experiences. In such applications, advanced content representations are required in order to increase the engagement of the user with the displayed imageries. Point clouds have emerged as a promising solution to this aim, due to their efficiency in capturing, storing, delivering and rendering of $3 \mathrm{D}$ immersive contents. As in any type of imaging, the evaluation of point clouds in terms of visual quality is essential. In this paper, benchmarking results of the state-of-the-art objective metrics in geometry-only point clouds are reported and analyzed under two different types of geometry degradations, namely Gaussian noise and octreebased compression. Human ratings obtained from two subjective experiments are used as the ground truth. Our results show that most objective quality metrics perform well in the presence of noise, whereas one particular method has high predictive power and outperforms the others after octree-based encoding.
\end{abstract}

\section{INTRODUCTION}

Recent trends in multimedia technologies indicate a significant growth of interest for new imaging modalities that aim to improve the user immersion capability. Among alternatives, point clouds denote a practical content representation that allows users to visualize static or dynamic scenes in a more immersive way. The interest for this richer type of visual modality has been renewed by the recent launch of low-cost depth sensors and their integration in hand-held devices, such as smartphones and laptops. An additional advantage of point clouds is that they offer a suitable format that can be directly exploited in systems that aim to provide immersive experiences with higher degrees-of-freedom. This feature makes this type of imaging a suitable candidate for visual data representation in augmented, mixed and virtual reality applications.

In such emerging technologies, the quality of user's experience is crucial and strongly related to the perceptual quality. The visual quality of a content is typically assessed through either subjective or objective quality assessment. Subjective evaluations are time consuming and expensive; thus, efficient objective metrics that can accurately predict the level of visual impairment of a distorted content are required. In the case of point clouds, objective quality assessment is still an open problem. The current objective metrics for position errors are based on full-reference approaches, which measure either the geometry or the normal similarity between the original and the distorted content, resulting in an error value that corresponds to the expected level of impairment. Considering the entire pipeline from acquisition and compression to rendering, point clouds are subject to various types of distortions of radically different nature. Recently, a significant amount of effort has been reported in the literature, investigating the performance of current objective metrics for point clouds under different types of distortions and visualization approaches.

Mekuria et al. [1] collected subjective scores for dynamic colored point clouds that were captured in real-time by multiple Microsoft Kinect sensors. The acquired models represented the avatars of the subjects that were able to navigate in a virtual environment in a desktop setup. The performance of the proposed codec that was deployed to encode these dynamic sequences was subjectively assessed in this $3 \mathrm{D}$ tele-immersive system. This codec was also assessed in the framework of the recent activities of the MPEG standardisation committee [2]. As a result of these efforts, benchmarking results are provided in [3], including bitrates and objective scores achieved for a set of static and dynamic colored point cloud contents. The subjective evaluation methodology followed by the MPEG group is also briefly described, although correlation results between subjective and objective scores are not reported. Javaheri et al. [4] subjectively evaluated the efficiency of point cloud denoising algorithms applied on contents distorted by Gaussian noise of different levels. To visualize the denoised contents, the Screened Poisson surface reconstruction [5] was used and the resulted 3D meshes were captured by different viewpoints with fixed distance between the virtual camera and the test contents. Animated videos were formed and rated by human observers in a flat monitor. In [6], the same authors conducted subjective quality assessment of colored point clouds, whose geometry was compressed under octreeand graph-based encoding schemes, while the original color attributes remained uncompressed. Both inanimate objects and human figures were selected and encoded in three quality levels. The contents under evaluation were visualized using cubes as primitives, whose size was determined as a function of local intrinsic resolutions. A virtual camera captured the model from different viewing angles following a spiral path. A video sequence was produced from these acquired instances, and was assessed by the subjects in a passive way in a desktop setting. In [7], [8], we proposed an interactive way 
for subjective quality assessment of point cloud structure using Double-Stimulus Impairment Scale (DSIS) and Absolute Category Rating (ACR) test methods, respectively, in a desktop setup. In both cases, two different types of distortions were evaluated: the first to simulate position errors from sensor inaccuracies, and the second to account for octree-based compression artifacts, while the contents were displayed as sets of points. In the second study, comparison between the DSIS and the ACR test methods is also reported. In [9], we proposed the use of head-mounted displays for subjective quality assessment of point clouds in an augmented reality scenario.

In these studies, though, only a subset of the available algorithms for objective quality assessment of point clouds is tested. In this paper, we describe every objective metric that has been considered in the recent efforts of the standardization bodies and in particular in JPEG. Benchmarking results are extensively reported using as ground truth subjective scores from two experiments that follow different evaluation methodologies, in order to assess the visual quality of colorless point clouds displayed as sets of points and subject to two different types of degradation.

\section{OBJECTIVE QUALITY ASSESSMENT OF POINT CLOUDS}

Objective quality assessment of point clouds is mainly carried out by full-reference metrics, which implies that both the distorted and the original contents should be available to compute a representative degradation value. The stateof-the-art objective metrics for geometric distortions can be distinguished into two main categories: (a) distance-based, and (b) normal-based. The normal-based metric, currently, consists of only one method, which is annotated as planeto-plane. The distance-based metrics can be further classified in three classes: (i) point-to-point, (ii) point-to-plane, and (iii) point-to-mesh [10]. The point-to-mesh metrics are based on projected distances between the point cloud under evaluation and the reconstructed reference object. Considering that there is no unique way to generate a mesh object from a set of points, the objective scores heavily depend on the selected surface reconstruction algorithm. Thus, point-to-mesh metrics are considered as sub-optimal solution for quality assessment of point clouds, and will not be further investigated in this study.

The point-to-point metric depends on geometric distances of associated points between the reference and the content under evaluation. In particular, following the notations of Figure 1, for each point $b_{k}$ of the content under evaluation $B$, its nearest neighbor $a_{i}$ from the reference point cloud $A$ is determined. Then, the Euclidean distance between them, $E\left(a_{i}, b_{k}\right)$, is calculated based on Equation 1.

$$
E\left(a_{i}, b_{k}\right)=\left\|\vec{v}_{a_{i}}^{b_{k}}\right\|_{2}
$$

Thus, the error value assigned to a distorted point reflects the geometric deviation from the original position, defined by the coordinates of the corresponding nearest reference point.

The point-to-plane metric [11] is based on the projection of the vector that connects two associated points, along the

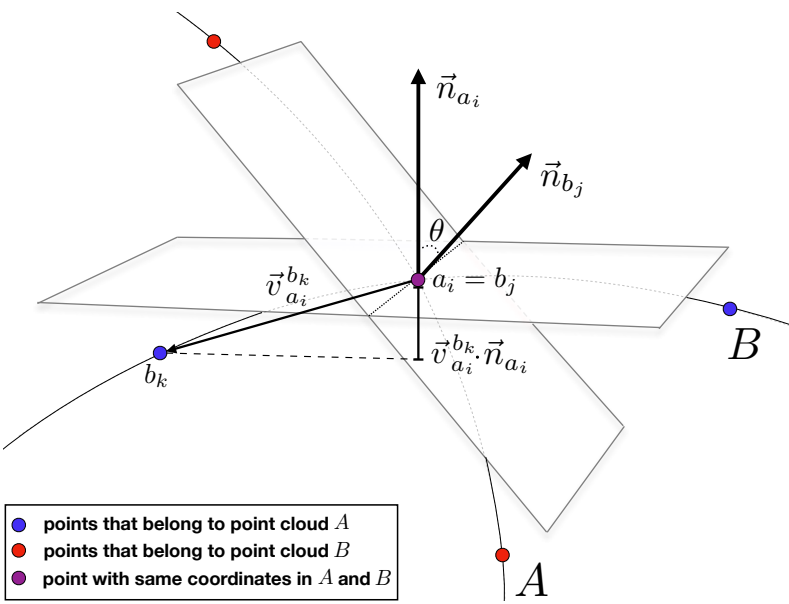

Fig. 1: Distances used by current objective metrics.

normal vector of the reference point. Specifically, after identifying for each point $b_{k}$ of the content under evaluation $B$ its nearest neighbor $a_{i}$ from the reference point cloud $A$, the projected error $\hat{E}\left(a_{i}, b_{k}\right)$ is calculated across the normal $\vec{n}_{a_{i}}$ of the reference point $a_{i}$, based on Equation 2 .

$$
\hat{E}\left(a_{i}, b_{k}\right)=\vec{n}_{a_{i}} \cdot \vec{v}_{a_{i}}^{b_{k}}
$$

The interpretation behind this metric is that larger costs are assigned to points that deviate from the underlying local surface that the reference point cloud approximates. However, the normal vectors of the original content are required. If the normals exist and the original point cloud is set as the reference, the computation is straight forward. If the distorted point cloud is set as the reference, its normals are estimated by averaging over the normals of associated nearest neighborhoods that belong to the original content. Then, the projected errors are estimated across these estimated average normals.

The plane-to-plane metric [12] is based on the angular similarity of tangent planes that correspond to associated points between the reference and the content under evaluation. For every point $b_{j}$ that belongs to the content under evaluation $B$, its nearest neighbor $a_{i}$ from the reference point cloud $A$ is identified. Then, the angular similarity of tangent planes can be computed from the normal vectors that correspond to these points. In particular, the angle $\hat{\theta}$ between the normal vectors $\vec{n}_{a_{i}}$ and $\vec{n}_{b_{j}}$ is firstly calculated. Then, since the angular similarity between the tangent planes is required, $\theta=\min \{\hat{\theta}, \pi-\hat{\theta}\}$ is computed, which corresponds to the minimum out of the two angles that are formed by the intersecting tangent planes, as shown in Figure 1. The angular similarity bounded in the range $[0,1]$ is given by Equation 3 .

$$
\text { Angular similarity }=1-\frac{2 \theta}{\pi}
$$

This metric is based on the consideration that the human visual system naturally interpolates a set of displayed points in order to infer the underlying object. The tangent plane serves as a linear approximation to the local surface of a content. Thus, the angular similarity between tangent planes of associated 
points between the original and the distorted contents, provides a coarse approximation of the dissimilarity between corresponding local surfaces of the underlying objects. However, this metric requires the normals of both the original and the distorted point clouds. If missing, the normal vectors have to be estimated. Thus, the performance of this metric is affected by the normal estimation algorithm and its configuration.

In every metric, each point of the content under evaluation is associated with an individual error, calculated through a corresponding equation, as given above. The level of degradation of a test content with respect to its original version is expressed through a total error value that can be estimated either as the Root Mean Squared (RMS) or the Mean Squared Error (MSE) or a simple average of the individual errors, or by taking the Hausdorff distance ${ }^{1}$. Different weights could be potentially assigned to the individual errors. Considering the distancebased metrics, such absolute values may lead to meaningless results for point cloud contents of different dimensions. To reduce the impact of different scales, the Peak-to-Signal Noise Ratio (PSNR) is proposed. In the literature, it is defined as the ratio of the squared maximum distance of the nearest neighbours of the original content (potentially, multiplied by a scalar), or the squared distance of the diagonal of the minimum bounding box, divided by the squared error value (i.e., squared RMS, MSE, squared average, or squared Hausdorff). Finally, for both distance- and normal-based metrics, a symmetric error is commonly used. This is obtained after setting both the original and the distorted contents as reference, and keeping the maximum out of both total errors.

\section{Methodology}

In this section we describe the content preparation, the subjective experiments, the details for the computation of the objective scores, and the methodology that was followed to benchmark the objective metrics. Further information regarding the generation of contents, the types of degradations, the methodologies and the equipment that was used in our tests can be found in [8].

\section{A. Content preparation}

The objective of this study is to benchmark objective quality metrics for point cloud structure. Thus, no color values were assigned to the points to avoid biases that may occur by their usage. As complex scenes are difficult to perceive by human subjets in the absence of color, a representative data set of five simple geometry-only point clouds was selected. In particular, cube and sphere were artificially generated, vase was manually scanned, and bunny and dragon were selected from the Stanford 3D Scanning Repository ${ }^{2}$. The contents were scaled to be fitted in a minimum bounding box of size 1 , and their point density was set in the same order of magnitude.

Two different types of degradations were selected, namely,

(i) Gaussian noise to model position errors due to sensor

\footnotetext{
${ }^{1}$ The Hausdorff distance is defined as the maximum of the distances of each point in one set from its nearest neighbor in the other set.

${ }^{2} \mathrm{http} / / /$ graphics.stanford.edu/data/3Dscanrep/
}

imprecisions, and (ii) compression based on octree decomposition that leads to sparser versions with structural loss. For Gaussian noise, the coordinates of every point is displaced along every $X, Y$ and $Z$ axis, according to a target standard deviation $\sigma=\{0.0005,0.002,0.008,0.016\}$. For octree-based compression, a suitable level-of-details is selected per content to maintain a target percentage of remaining points $p=$ $\{30 \%, 50 \%, 70 \%, 90 \%\}$ with acceptable deviation of $\pm 2 \%$. This type of distortion is annotated as octree-pruning.

In Figure 2, two of the selected original test contents along with a corresponding degraded version after Gaussian noise and octree-pruning, are indicatively illustrated.
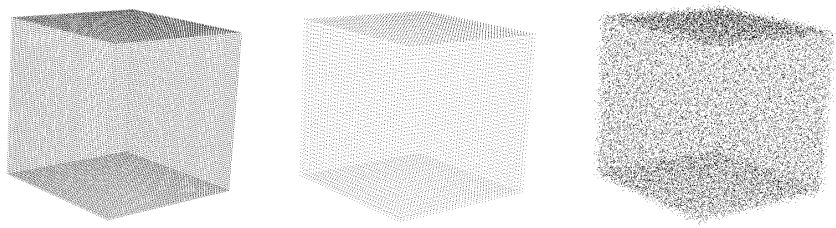

(a) Cube: (left) original, (middle) octree-pruning with $p=50 \%$, (right) Gaussian noise with $\sigma=0.008$.
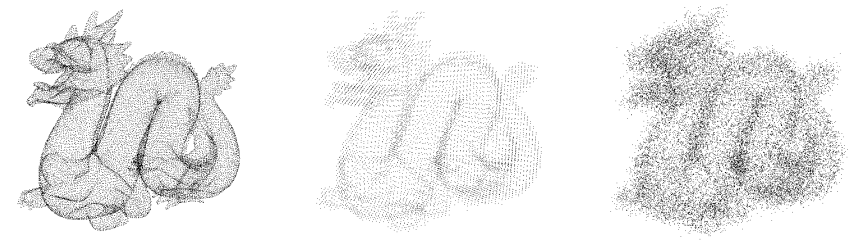

(b) Dragon: (left) original, (middle) octree-pruning with $p=50 \%$, (right) Gaussian noise with $\sigma=0.008$.

Fig. 2: Illustration of artifacts in degraded test contents.

\section{B. Subjective quality assessment as ground truth}

The experiments were conducted in a laboratory that fulfills the ITU-R Recommendation BT.500-13 [13] for subjective evaluation of visual data. A visualizer based on the Point Cloud Library (PCL) [14] was used in an Apple Cinema Display of 30 inch (res. 2560x1600). The subjects were able to visualize the test contents as sets of points on the flat screen, interact using the mouse cursor, and provide their scores using the keyboard. Two different subjective evaluation methodologies were selected (i) simultaneous DSIS, and (ii) ACR, with 5-grade impairment scale and a hidden reference. The first is preferred for its high discriminative power, whereas the second simulates realistic consumption of visual data.

As two different types of degradations introducing different visible distortions were selected, the experiment was split in 4 sessions. In each session, a training phase took place in order for the subjects to get familiar with the artifacts under assessment and the interaction part. For the actual evaluations, different permutations of the order of contents were deployed, the same content was never displayed consecutively, and the side of the reference in the screen was selected randomly per subject for the DSIS methodology. Every session was 
TABLE I: Performance indexes of objective metrics against ground truth subjective scores (bold text indicates best performance).

\begin{tabular}{|c|c|c|c|c|c|c|c|c|c|c|c|c|c|c|c|c|}
\hline \multirow[b]{3}{*}{ Metric } & \multicolumn{8}{|c|}{ DSIS test method } & \multicolumn{8}{|c|}{ ACR test method } \\
\hline & \multicolumn{4}{|c|}{ Gaussian noise } & \multicolumn{4}{|c|}{ Octree-pruning } & \multicolumn{4}{|c|}{ Gaussian noise } & \multicolumn{4}{|c|}{ Octree-pruning } \\
\hline & PCC & SROCC & RMSE & OR & PCC & SROCC & RMSE & OR & PCC & SROCC & RMSE & OR & PCC & SROCC & RMSE & OR \\
\hline po2 point $_{\text {RMS }}$ & 0.9949 & 0.9488 & 0.1404 & 0.05 & 0.1524 & -0.1499 & 0.9403 & 0.70 & 0.9867 & 0.9326 & 0.2232 & 0.25 & 0.3698 & -0.0045 & 0.8497 & 0.60 \\
\hline po2plane $\mathrm{RMS}$ & 0.9845 & 0.8969 & 0.2430 & 0.25 & 0.1835 & -0.2004 & 0.9352 & 0.70 & 0.9811 & 0.9003 & 0.2662 & 0.25 & 0.3939 & -0.0467 & 0.8406 & 0.60 \\
\hline po2point $\mathrm{MSE}$ & 0.9605 & 0.9391 & 0.3858 & 0.60 & 0.1670 & -0.1763 & 0.9380 & 0.70 & 0.9561 & 0.9266 & 0.4025 & 0.55 & 0.3776 & -0.0286 & 0.8468 & 0.55 \\
\hline po2plane $\mathrm{MSE}$ & 0.9357 & 0.9278 & 0.4893 & 0.70 & 0.1965 & -0.1748 & 0.9329 & 0.70 & 0.9360 & 0.9379 & 0.4836 & 0.70 & 0.4028 & -0.0211 & 0.8371 & 0.55 \\
\hline po2point Hausdorff & 0.9936 & 0.9353 & 0.1569 & 0.10 & 0.2473 & 0.2525 & 0.9219 & 0.55 & 0.9864 & 0.9304 & 0.2260 & 0.15 & 0.2540 & -0.0956 & 0.8846 & 0.60 \\
\hline po2plane ${ }_{\text {Hausdorff }}$ & 0.9932 & 0.9368 & 0.1609 & 0.10 & 0.2294 & 0.1928 & 0.9260 & 0.60 & 0.9873 & 0.9304 & 0.2181 & 0.20 & 0.2871 & -0.0421 & 0.8761 & 0.60 \\
\hline PSNR_po2point $_{\text {RMS }}$ & 0.9948 & 0.9707 & 0.1416 & 0.10 & 0.2840 & 0.2765 & 0.9122 & 0.50 & 0.9930 & 0.9846 & 0.1629 & 0.10 & 0.2207 & 0.1415 & 0.8920 & 0.65 \\
\hline PSNR_po2plane $_{\text {RMS }}$ & 0.9827 & 0.9360 & 0.2568 & 0.30 & 0.2854 & 0.2719 & 0.9118 & 0.50 & 0.9834 & 0.9469 & 0.2492 & 0.25 & 0.2873 & -0.1061 & 0.8760 & 0.60 \\
\hline PSNR_po2point $_{\text {MSE }}$ & 0.9950 & 0.9707 & 0.1391 & 0.10 & 0.2839 & 0.2765 & 0.9123 & 0.50 & 0.9936 & 0.9846 & 0.1555 & 0.10 & 0.2212 & 0.1415 & 0.8919 & 0.65 \\
\hline PSNR_po2plane $\mathrm{MSE}$ & 0.9947 & 0.9707 & 0.1422 & 0.05 & 0.2825 & 0.2855 & 0.9126 & 0.50 & 0.9950 & 0.9846 & 0.1372 & 0.10 & 0.2146 & 0.1332 & 0.8933 & 0.65 \\
\hline PSNR_po2point Hausdorff & 0.9960 & 0.9782 & 0.1231 & 0.05 & 0.3364 & 0.4384 & 0.8960 & 0.60 & 0.9945 & 0.9846 & 0.1435 & 0.15 & 0.2291 & 0.2942 & 0.8902 & 0.65 \\
\hline PSNR_po2plane ${ }_{\text {Hausdorff }}$ & 0.9959 & 0.9782 & 0.1253 & 0.05 & 0.2997 & 0.3420 & 0.9077 & 0.55 & 0.9951 & 0.9846 & 0.1361 & 0.15 & 0.2223 & 0.1934 & 0.8917 & 0.65 \\
\hline pl2plane Average & 0.9645 & 0.9466 & 0.3660 & 0.35 & 0.8925 & 0.9032 & 0.4292 & 0.35 & 0.9676 & 0.9665 & 0.3468 & 0.25 & 0.9530 & 0.9353 & 0.2771 & 0.15 \\
\hline pl2plane $\mathrm{RMS}$ & 0.9701 & 0.9466 & 0.3365 & 0.35 & 0.8868 & 0.9040 & 0.4397 & 0.35 & 0.9736 & 0.9665 & 0.3134 & 0.25 & 0.9536 & 0.9398 & 0.2754 & 0.15 \\
\hline pl2plane MSE & 0.9696 & 0.9466 & 0.3392 & 0.35 & 0.8856 & 0.9040 & 0.4419 & 0.30 & 0.9731 & 0.9665 & 0.3166 & 0.30 & 0.9528 & 0.9398 & 0.2776 & 0.15 \\
\hline
\end{tabular}

completed by 20 subjects ranging from 21 to 37 years of age and an average of 28 . A total of 5 contents and 4 degradation values were used along with a hidden reference, resulting in 25 stimuli per session. An outlier detection algorithm based on the ITU-R Recommendation BT.500-13 [13] was issued on the collected subjective scores. In DSIS with octree-pruning, one outlier was found resulting in 19 out of 20 , while in the remaining sessions no outliers were found (i.e., 20 out of 20 scores). After discarding the scores of the outliers, the Mean Opinion Scores (MOS) and the corresponding confidence intervals assuming a Student's t-distribution were computed. The average total time of interaction of the users with a content under assessment found to be 10.17 seconds with a standard deviation of 12.11, without observing notable differences for the time spent per content between the sessions.

\section{Computation of the objective scores}

The original contents of this dataset had no associated normals with their coordinates. To compute the normals, the regression method proposed by Hoppe et al. [15] was selected, as implemented in PCL [14], setting 6 nearest neighbors around each point of interest. The objective scores from every metric were calculated based on the same contents. The software described in [10] was used for the distance-based metrics. In particular, for the total error values based on the RMS and the Hausdorff distance, the implementation of the version 0.02 was adopted, while the version 0.09 was used to compute the error based on the MSE. The corresponding PSNR values, defined as the ratio of the squared maximum distance of nearest neighbours of the original content divided by the squared error value, were also considered for both point-to-point and point-to-plane metrics. The normal-based metric was computed following the implementation of [12]. In addition to the average angular similarity, the total error based on the RMS and the MSE is also measured.

\section{Benchmarking of objective metrics}

To evaluate how well an objective metric is able to predict the perceptual quality, the MOS obtained from subjective experiments are taken as ground truth and compared to predicted
MOS values obtained from objective metrics. The result of execution of a particular objective metric defines a Point cloud Quality Rating (PQR). A predicted MOS, denoted as $\operatorname{MOS}_{P}$ which corresponds to a specific distorted content, is estimated after a regression analysis on each [PQR, MOS] pair. The monotonic cubic function was used as a regression model, and the predicted MOS was computed as $\operatorname{MOS}_{p}=$ $a \cdot x^{3}+b \cdot x^{2}+c \cdot x+d$, where $a, b, c$ and $d$ were determined using a least squares method.

Based on Recommendation ITU-T P.1401 [16], to assess the performance of the objective metrics with respect to the ground truth subjective scores, the Pearson linear correlation coefficient (PCC), the Spearman rank order correlation coefficient (SROCC), the root-mean-square error (RMSE), and the outlier ratio (OR) based on standard error were computed, in order to examine the linearity, the monotonicity, the accuracy, and the consistency of the results, respectively.

\section{RESUlTS AND DISCUSSIONS}

In Table I, the performance indexes of the state-of-the-art objective metrics are reported for both subjective evaluation methodologies and both types of degradation. In Figures 3 and 4 , we present the scatter plots of the subjective against the objective scores from the best-performing metrics for the DSIS and the ACR experiments, respectively, along with the cubic fitting curve. Please note that the point-to-point, pointto-plane and plane-to-plane metrics are marked as po2point, po2plane and pl2plane, respectively.

Based on our results, every distance-based metric performs quite well in predicting the visual quality of distorted contents in the presence of Gaussian noise. Considering that these metrics capture geometric distances of closest points between the reference and the processed contents, by increasing the standard deviation of the noise, the objective scores worsen. The subjects are naturally able to recognize such distortions and identify the amount of noise introduced by the level of points' displacement. The normal-based metric performs slightly worse, achieving, though, a comparable performance. 


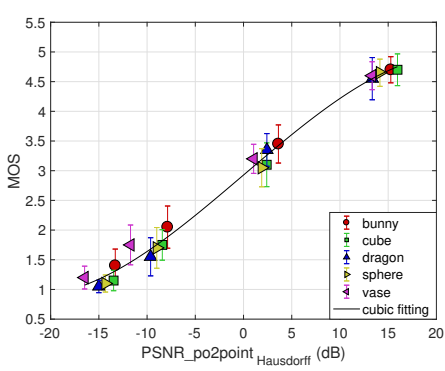

(a) Gaussian noise

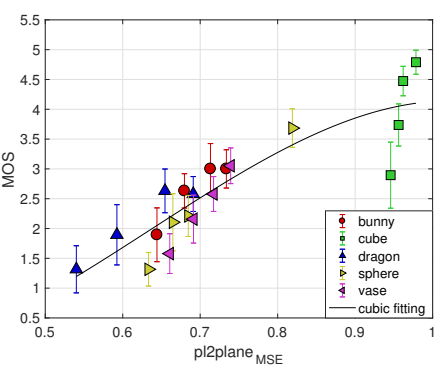

(b) Octree-pruning

Fig. 3: Scatter plots using DSIS test method.

In the presence of octree-based compression artifacts, the correlation between subjective and objective scores is poor for every distance-based objective metric. These methods capture the position errors occurring after the downsampling that takes place in the distorted content. However, the visual quality does not follow the same trend. In general, this type of compression leads to elimination of high frequency components and the perception of structural loss. Thus, the visual quality of point clouds with high curvature values and irregular structures is more severely impacted, while for low curvature, regular geometry contents, the structure is not significantly affected by such distortions, thus leading to less perceptible visual degradations. On the other hand, the correlation between the subjective and the predicted MOS obtained using every planeto-plane variation is quite strong. Considering that in the absence of noise, higher quality normal estimation is achieved, a normal-based metric seems to be a better approach, as it predicts the visual impairments based on an approximation of the dissimilarity between local surfaces of the inferred models.

\section{CONCLUSiOnS}

In this paper the state-of-the-art objective metrics are benchmarked using a set of colorless point clouds with diverse geometric structure. Results show that, to achieve high predictive power, different objective metrics should be used for different types of distortions. In particular, the visual quality of contents in the presence of noise, modelling sensor imprecisions, can be better predicted by distance-based metrics. Conversely, the perceptual degradations occurring in presence of compressionlike artifacts are better correlated with predicted MOS from normal-based methods. These results imply the necessity for more adequate solutions, which potentially incorporate both approaches, that would maintain high predictive power in the presence of every type of distortion. Even though the number and the geometric characteristics of these contents may be considered as representative for small-scale geometryonly point clouds, different performance might be observed for other contents and types of artifacts. Furthermore, the performance of these metrics is affected by the selection and the configuration of the normal estimation algorithm.

\section{ACKNOWLEDGEMENT}

This work has been conducted in the framework of ImmersiaTV under the European Unions Horizon 2020 research

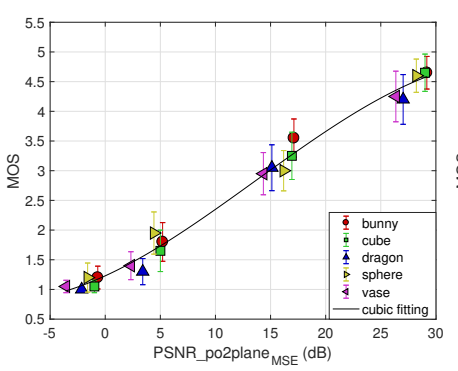

(a) Gaussian noise

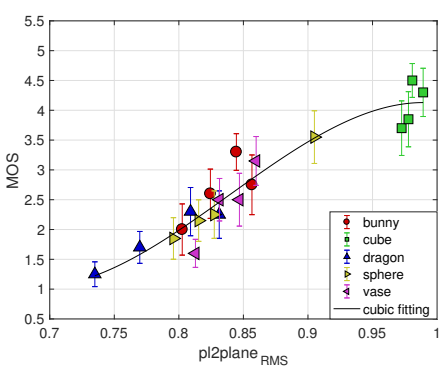

(b) Octree-pruning
Fig. 4: Scatter plots using ACR test method.

and innovation programme (grant agreement no. 688619) funded by Swiss State Secretariat for Education, Research and Innovation SERI.

\section{REFERENCES}

[1] R. Mekuria, K. Blom, and P. Cesar, "Design, Implementation, and Evaluation of a Point Cloud Codec for Tele-Immersive Video," IEEE Transactions on Circuits and Systems for Video Technology, vol. 27, no. 4, pp. 828-842, Apr. 2017.

[2] MPEG 3DG and Req., "Call for proposals for Point Cloud Compression V2," ISO/IEC MPEG2017/N16763, Hobart, AU, Apr. 2017.

[3] R. Mekuria, S. Laserre, and C. Tulvan, "Performance assessment of point cloud compression," in 2017 IEEE Visual Communications and Image Processing (VCIP), Dec. 2017, pp. 1-4.

[4] A. Javaheri, C. Brites, F. Pereira, and J. Ascenso, "Subjective and objective quality evaluation of 3D point cloud denoising algorithms," in 2017 IEEE International Conference on Multimedia Expo Workshops (ICMEW), Jul. 2017, pp. 1-6.

[5] M. Kazhdan and H. Hoppe, "Screened Poisson Surface Reconstruction," ACM Trans. Graph., vol. 32, no. 3, pp. 29:1-29:13, Jul. 2013.

[6] A. Javaheri, C. Brites, F. Pereira, and J. Ascenso, "Subjective and objective quality evaluation of compressed point clouds," in 2017 IEEE 19th International Workshop on Multimedia Signal Processing (MMSP), Oct. 2017, pp. 1-6.

[7] E. Alexiou and T. Ebrahimi, "On subjective and objective quality evaluation of point cloud geometry," in 2017 Ninth International Conference on Quality of Multimedia Experience (QoMEX), May 2017, pp. 1-3.

[8] — - "On the performance of metrics to predict quality in point cloud representations," in Proceedings of SPIE, ser. Applications of Digital Image Processing XL, vol. 10396, Aug. 2017.

[9] E. Alexiou, E. Upenik, and T. Ebrahimi, "Towards subjective quality assessment of point cloud imaging in augmented reality," in 2017 IEEE 19th International Workshop on Multimedia Signal Processing (MMSP), Oct. 2017, pp. 1-6.

[10] D. Tian, H. Ochimizu, C. Feng, R. Cohen, and A. Vetro, "Evaluation Metrics for Point Cloud Compression,” ISO/IEC MPEG2016/M39316, Chengdu, China, Oct. 2016.

[11] _ - "Geometric distortion metrics for point cloud compression," in 2017 IEEE International Conference on Image Processing (ICIP), Sep. 2017, pp. 3460-3464.

[12] E. Alexiou and T. Ebrahimi, "Point cloud quality assessment metric based on angular similarity," in 2018 IEEE International Conference on Multimedia and Expo (ICME), Jul. 2018, pp. 1-6.

[13] ITU-R BT.500-13, "Methodology for the subjective assessment of the quality of television pictures," International Telecommunications Union, Jan. 2012.

[14] R. B. Rusu and S. Cousins, "3D is here: Point Cloud Library (PCL)," in 2011 IEEE International Conference on Robotics and Automation, May 2011, pp. 1-4.

[15] H. Hoppe, T. DeRose, T. Duchamp, J. McDonald, and W. Stuetzle, "Surface Reconstruction from Unorganized Points," in Proceedings of the 19th Annual Conference on Computer Graphics and Interactive Techniques, ser. SIGGRAPH '92. ACM, Jul. 1992, pp. 71-78.

[16] ITU-T P.1401, "Methods, metrics and procedures for statistical evaluation, qualification and comparison of objective quality prediction models," International Telecommunication Union, Jul. 2012. 